\title{
ICU Practice
}

\section{Blood Culture Techniques: Increasing Yield and Reducing Contamination}

\author{
Terrence Rohan Chinniah* \\ *Faculty of Medicine, University of Colombo, Sri Lanka
}

\begin{abstract}
Bloodstream infections are associated with significant patient mortality and health care costs. Isolation of the causative organism will direct the clinician to institute specific treatment. But isolation of the implicating organism is hampered by lack of yield and increased level of contamination. The rates of contamination and reduced yield are more relevant in Sri Lanka as most of our public sector laboratories use manual blood culture system and do not adhere to correct aseptic techniques of blood collection. This has led to delay in identifying the causative organism. Increase rates of contamination not only have hindered specific treatment but also have prompted clinicians to use antibiotics of increased cost and toxicity. Though differentiation between true pathogen and contamination is not clearly set, certain parameters, namely identification of the organism, proportion of the positive blood culture sets, Number of positive blood culture bottles within a blood culture set, time to growth, source of cultures (catheter drawn versus percutaneous) along with clinical and laboratory clues might help the clinicians and microbiologist to make a reasonable conclusion. Proper skin preparation with correct disinfectants, adherence to recommended techniques and contact time along with the collection of optimal volume of blood through a peripheral vein by a trained phlebotomy team has been found to increase the yield and reduce the rates of contamination. These recommendations could be practiced with ease in resource poor settings similar to Sri Lankan public sector hospitals.
\end{abstract}

\section{Introduction}

Blood cultures are the most important laboratory tests performed in the diagnosis of serious infections, and would lead to a definitive diagnosis and therapy against the causative organism ${ }^{1}$. But ambiguous results and contamination among blood cultures has become apparent, costly, and confusing the clinicians $2,3,4,5,6$.

Though target rates for contamination have been set at 2 to $3 \%$, actual rates vary between $0.6 \%$ to $6 \% 7$. A gold standard to differentiate true pathogen from contaminant / organisms of unknown significance do not exist, but clinical studies of bloodstream infections may help $6,8,9,10$. Despite its limitations, the blood culture remains the "gold standard" for the detection of bacteremia.

Microbiologists and clinicians use a laboratory and clinical tools in deciding whether a blood isolate is a pathogen or a contaminant 11 . These include 1 ) the identity of the microorganism 2) clinical features such as fever, leukocytosis, level of Creactive protein and results of imaging studies 3) the proportion of blood culture sets positive against the number of sets obtained 4) the time it takes for growth to be detected 5) the number of culture bottles within a culture set that show growth.

Microorganisms that always or nearly always (>90\%) represent true bacteremia or fungemia include Staphylococcus aureus, Streptococcus pneumoniae, Escherichia coli and other members of the Enterobacteriaceae, Pseudomonas aeruginosa, and Candida albicans ${ }^{10 .} \quad$ Streptococcus pyogenes, Streptococcus agalactiae, Listeria monocytogenes, Neisseria meningitidis,

Correspondence: errence Rohan Chinniah Faculty of Medicine, University of Colombo, 25, Kynsey Road, Colombo 08, Sri Lanka. Email: trctrc@gmail.com 
Neisseria gonorrhoeae, Haemophilus influenzae, Bacteroides fragilis group, Candida species other than C. albicans, and Cryptococcus neoformans usually represent true infection ${ }^{11 .}$

Coagulase-negative staphylococci (CoNS) which were previously considered as contaminants, have increased in clinical importance and are now recognized as pathogens as the etiologic agents of catheter-associated bacteremia and bacteremia in patients with vascular and other prostheses and judging its clinical significance is very challenging ${ }^{10,12,13}$.

Hence an accurate interpretation of culture results is critical for the perspective of individual patient care as well in the tracking and reporting of nosocomial infections and monitoring of bloodstream infection rates, which depend mostly if not totally on the accurate differentiation of contamination from true bacteremia.

\section{Interpretation of Blood culture} Identity of Organism

Seventy nine percent (79\%) of the participating laboratories in the CAP QProbes study cited identity of the organism as the most important indicator to interpret blood culture results ${ }^{14}$. Also Bates et al. found that the identity of the organism was the most important predictor in differentiating contaminated blood culture15.

Weinstein et al. suggested that presence of certain organisms, namely Staphylococcus aureus, Streptococcus pneumoniae, Escherichia coli and other Enterobacteriaceae, Pseudomonas aeruginosa, and Candida albicans should almost always be thought to represent true bacteremia or fungemia when isolated from a blood culture ${ }^{10}$. Certain organisms, namely coagulase-negative staphylococci, Corynebacterium species, Bacillus species other than Bacillus anthracis, Propionibacterium acnes, Micrococcus species, viridans group streptococci, enterococci, and Clostridium perfringens have been found to represent contamination in a significant proportion of cases $^{10,11}$. However, it is crucial to recognize that these organisms can also cause true bacteremia with devastating consequences, if untreated due to misinterpretation as contaminants 7 .

Though, coagulase-negative staphylococci were usually believed to represent contamination in the past typically representing $70 \%$ to $80 \%$ of all contaminated blood cultures $14,16,17,18$, recent studies have shown that these organisms being identified as source of true bacteremia in patients with prosthetic devices and central venous catheters ${ }^{13,14}$.

Traditionally bloodstream infections are thought to involve only a single organism, prompting clinicians to conclude growth of multiple organisms from a blood culture bottle as contaminated. Shafazand et al found that $6 \%$ to $21 \%$ of all true bacteremia are polymicrobial, usually in high-risk patient groups ${ }^{19}$.

\section{Proportion of Positive Blood Culture Sets}

The proportion of positive sets compared to that of the total number of sets obtained can be a useful tool $8,20,21,22$. To consider a growth as true bacteremia, multiple blood culture sets will usually grow the same organism showing an improvement in the positive predictive value ${ }^{20}$. The presence of only one positive set among at least two sets drawn at the same time may be indicative of culture contamination and also if only one set grows an organism known to cause contamination, it could be considered to be a contamination although it may conversely indicate transient bacteremia? In order to differentiate transient bacteremia from contamination, it has been recommended that multiple sets of blood cultures (at least two sets) of cultures be obtained at the same time to recognize potential bloodstream infections $8,23,24$.

\section{Number of Positive Blood Culture Bottles} within a Blood Culture Set

Though controversial, the number of blood culture bottles that exhibit growth within a given blood culture set has been used to differentiate contaminated blood cultures from cultures that represent bacteremia ${ }^{25}$, 26. If only one bottle exhibits growth within a given set, the likelihood of contamination is thought to be greater ${ }^{26}$. 
Usually, a set of blood cultures involves one aerobic bottle and one anaerobic bottle in an attempt to optimize the isolation of both aerobic and anaerobic organisms. This may not be applicable in Sri Lanka as neither government nor private sector use anaerobic bottle.

\section{Time to Growth / Positivity}

The amount of time required for the organism to grow in the culture medium is thought to be helpful in differentiating contamination. Conceptually, it is thought that the blood from a bacteremic patient will have a much higher inoculum of bacteria than a contaminated culture ${ }^{7}$. Hence the detection of growth is earlier than a smaller inoculum ${ }^{27,28}$. Several studies have shown that cultures that become positive more than 3 to 5 days after incubation have been more likely to represent contaminants ${ }^{28,} 29$. The difficulty in obtaining more than one set of cultures in the pediatric population, prompted HaimiCohen et al. to used clinical parameters to differentiate true coagulase-negative infections from contaminants. They found that a time to positivity of $15 \mathrm{~h}$ had a positive predictive value of $84 \%$ for true infection in children ${ }^{30}$.

Although not useful as the identification of the organism or the presence of multiple positive cultures, Bates et al. found that the time to growth was a useful variable in a multivariate algorithm for predicting true bacteremia ${ }^{3}$. But as this parameter was not found to be useful by other studies some experts recommend that this technological variable should not be relied upon to distinguish contaminants from pathogens in blood cultures18.

\section{Clinical and Laboratory Clues}

Clinical criteria combined with laboratory data such as white cell count and neutrophil count, have been used in several studies to differentiate contaminated cultures from clinically significant cultures ${ }^{3}, 20,30,31$. Other surrogate markers for infection, including the use of C-reactive protein measurements

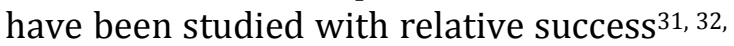
$33,34,35$.

\section{Source of Cultures (Catheter Drawn versus} Percutaneous)

A positive blood cultures drawn through a vascular catheter could indicate three possibilities: true bacteremia, catheter colonization, or culture contamination? Catheter colonization is not contamination, nor is it infection, which is contrast to the culture contamination is due to the organism that grow on the surface of a catheter forming a biofilm which may or may not progress to cause symptoms of infection or true bacteremia ${ }^{7} .15$ to $25 \%$ of short-term central venous catheters, usually get colonized by coagulase-negative staphylococci, with no evidence of infection ${ }^{36}, 37$. Therefore, cultures drawn from central lines could be positive due to colonization rather than true infection. Hence it is recommended that if cultures are obtained from a catheter, at least one set should be drawn percutaneously33, 38, 39, 40. This was proven by a mathematical modeling study by Tokars ${ }^{41}$.

\section{Strategies recommended to prevent contamination}

Skin preparation

Skin of the patient at the site of venepuncture is thought to be the most common source of contamination7. Since $20 \%$ of skin-associated bacteria have been found to survive disinfection, skin antisepsis cannot entirely prevent the contamination of blood cultures $42,43$. Though the skin bacteria that are located in deep layers of the skin or in other structures that antiseptics cannot penetrate may be responsible for contamination inadequate skin preparation is thought to be the most common cause of blood culture contamination ${ }^{44,45}$.

Mimoz et al found that $0.5 \%$ chlorhexidine plus alcohol had significantly lower contamination rates than standard povidone-iodine46. In contrast, when chlorhexidine was compared to tincture of iodine did not show statistically significant difference in contamination rates 47,48 . The most commonly studied and traditionally used skin antiseptic for blood culturing is povidone-iodine. Significantly low contamination rates of contamination were found on using iodine tincture compared 
with povidone-iodine $14,49,50$. But the use of iodine tincture has to be weighed against the cost in resource poor setting similar to Sri Lanka where alcohol-based products are also frequently utilized for skin preparation for blood cultures.

A randomized comparative trial done by Calfee and Farr of four skin antiseptics, $10 \%$ povidone iodine, $70 \%$ isopropyl alcohol, tincture of iodine, or povidone-iodine plus $70 \%$ ethyl alcohol showed the contamination rates were $2.93 \%$ with povidone-iodine, $2.58 \%$ with tincture of iodine, $2.50 \%$ with isopropyl alcohol, and $2.46 \%$ with povidone-iodine plus $70 \%$ ethyl alcohol respectively ${ }^{51}$. Although there were no significant differences between the groups, authors concluded that the antiseptics that contained alcohol may have had greater efficacy.

The time required for the antiseptic to have maximal effect is an important consideration, since the agent with the most efficacy will not be effective if the time required to become effective is longer than clinically feasible ${ }^{7}$. Some experts recommend that the culture site be prepared with $70 \%$ isopropyl or ethyl alcohol and allowed to air dry and followed by 1 to $2 \%$ tincture of iodine or $10 \%$ povidone-iodine 24 .

It is vital to realize that the contact time of the antiseptic is more important than the antiseptic used because organisms die by drying rather than drowning. Hence it is apparent that contamination may be more related to technique than the actual antiseptic used ${ }^{14}$.

The practice of re-palpating of the vein prior to venepuncture after skin disinfection should be avoided. It is important to realize venepuncture for blood culture is a sterile procedure unlike those for haematological or biochemical investigation.

\section{Culture bottle preparation}

The rubber stopper on each bottle is not sterile despite being covered with a lid that requires removal prior to inoculation and the standard practice to disinfect the tops of the culture bottles before inoculating them with blood ${ }^{14}$.
It has been recommended that iodine alone should not be used as it may cause erosion of the rubber stopper during incubation, thereby introducing contaminants. Alcohol alone is recommended to be used to disinfect the top of the bottle in Sri Lanka prior to inoculation of the blood culture bottles.

\section{Single needle versus double needle techniques}

Till the emergence of human immunodeficiency virus double-needle technique was standard practice ${ }^{7}$. In this technique the needle used to draw blood cultures is discarded and a new, different needle is used to inoculate the bottles. However, the process of changing needles was felt to increase the risk of needlestick injuries. Hence double-needle technique has been discouraged in favor of using the same needle to draw blood and to inoculate the culture bottles (single-needle technique) ${ }^{52}$, 53,54 .

Though a meta-analysis show a statistically significant difference as the double-needle technique did infact decrease contamination rates from $3.7 \%$ to $2.0 \%$ (P < $0.001)^{54}$ any benefit from the double-needle technique did not outweigh the risk of needlestick injuries ${ }^{7}$.

Obtaining cultures Percutaneously instead of via Vascular catheters

Many clinicians prefer to draw blood through a vascular catheter than percutaneous venepuncture to prevent inflicting pain, decrease the likelihood of inducing transient bacteremia by phlebotomy, especially for highly immunosuppressed patients and the difficulty of venous access among the neonatal population.

Despite these good intentions, the colonization of the organisms on these vascular catheters will lead to more cultures for clarification, more diagnostic studies, or the unnecessary use of antibiotics $7,36,37$.

It is always recommended to obtain blood for blood culture through a percutaneous venepuncture. 


\section{Trained phlebotomy team}

Concrete evidence have been brought forth by many studies to show that a dedicated phlebotomy team will significantly reduce contamination rate and increase the yield $11,55,56$ In the light of Q-Probe study which showed low contamination rates when blood for cultures were drawn by dedicated phlebotomy team compared to the other staff including resident physicians ${ }^{57}$, it will be reasonable to explore the possibility of having dedicated phlebotomists in Sri Lankan settings, which will prove to reduce the healthcare cost as has been shown by other studies ${ }^{58}$. Monitoring and feeding back contamination rates to individuals who obtain blood cultures were used successfully in at least one other institution that achieved a 50\% reduction in contamination rates using this methodology59. Similar monitoring and feedback used by author and colleague at an institution too showed encouraging rates of reduction in contamination from 14\% to $8 \%$ percent over a period of one year (unpublished data)

\section{Blood culture collection kits}

A home made collection kit including blood culture bottles, $20 \mathrm{ml}$ syringe, quick reference guidelines for collecting blood culture, alcohol and chlohexidine swipes along with monitoring and feed back, were used by author and colleagues have shown to reduce the contamination rates from 14 $\%$ to $11 \%$ within 2 months ${ }^{60}$. The contamination rates were further reduced to $8 \%$ over the next year (unpublished data). Similarly commercially available blood culture collection kits have shown to reduce contamination rates in some studies $^{58}$.

\section{Technique of blood culture collection, Storing and Transportation}

Selection of patients

Blood cultures are to be done when there are reasonable expectations of bacteremia or fungicidal hence patient selection is vitally important. Some of the parameters used in identifying the patients will be temperature of $>38^{\circ} \mathrm{C}$ or $<36^{\circ} \mathrm{C}$, hypotension, tachycardia, cold clammy extremities etc ${ }^{61}$.

\section{Timing of the blood culture}

Blood cultures should be drawn prior to the institution of antibiotics whenever possible. If empiric treatment is an emergency, blood cultures should still be drawn as soon as possible after institution of antibiotics.

Bennett and Beeson reported that the presence of micro organisms in the blood is the initiating event leading to fever with chill and rigors $1-2$ hours later 62 and blood cultures are negative at the time of temperature spike. But it is practically not possible to obtain a blood culture 1 hour before the onset of fever. Thomson and colleagues found that rates of bacteremia detection were not enhanced by collecting blood cultures at the time that patients were noted to have temperature spikes ${ }^{63}$.

Further Stefan et al concluded that is not necessary in routine practice to collect blood for culture at the time that adult patients are experiencing a temperature elevation64. Hence increasing the frequency and spreading out the sampling time will increase the yield 24 .

In suspected cases of continuous bacteremia, endocarditis, thromboplebitis timing of culture becomes irrelevant ${ }^{24}$. In those with intermittent bacterium organism may be identified by obtaining multiple blood sets with wide time interval. But this time interval in clinical practice is determined by the urgency to initiate antibiotic therapy.

In medically urgent situations e.g. meningitis febrile neutropenia, two to three sets of blood cultures could be collected from different peripheral veins at 30 minutes interval before initiating antibiotic.

\section{Optimal volume for collection}

The volume of the blood is the most important factor affecting the sensitivity of blood culture ${ }^{24}$. Ilstrup and Washington concluded that large volume of blood will significantly increase the yield of positive blood culture ${ }^{65}$. Based on their finding they recommended that $10 \mathrm{ml}$ of blood should be inoculated into each blood culture bottle. More than $30 \mathrm{ml}$ of blood will not increase the yield and precipitate nosocomial anaemia66. Since magnitude of bacteremia 
is greater among paediatric patients less amount of blood 0.5 to $3 \mathrm{ml}$, is required 67 .

The dilution of blood to medium is maintained between 1:5 to 1: 10 (ideally $1: 10)$ to dilute antimicrobial factors such as antibodies, complements phagocytes and antibiotics $^{24}$.

The blood volume and dilution factor has been recognized as a major factor affecting the yield in Sri Lanka. The traditionally McCartney (Universal) bottles are used in manual blood culture system. Since these bottles have an interior volume of $20 \mathrm{ml}$, only $3-4 \mathrm{ml}$ of blood can be inoculated if 1:5 dilution to be maintained. This will greatly affect the yield of positive culture. Further reusing of these bottles, manual preparation and dispensation of media into these bottles adds risk to culture contamination.

Storing and transportation to the laboratory It is always recommended that the blood culture bottles to be labeled prior to blood collection and the request form to be furnished with all relevant clinical details.

Once the blood is inoculated into blood culture bottle, proper mixing of the blood with the medium is vital. If not the blood will get clotted and the organism will be entrapped in the fibrin mesh of the clot preventing its multiplication in the blood culture medium. This will be more relevant for the manually prepared blood culture bottles.

If any delay in transportation of the blood culture bottles after inoculation is anticipated these bottles should left at room temperature. They should not be refrigerated as this could delay the multiplication of all organisms and certain organisms like Streptococcus pneumoniae, Haemophilus influenzae could be inhibited.

\section{Conclusion}

Bloodstream infections are an important cause of morbidity and mortality. Identification of the causative organism and prompt administration of appropriate antimicrobial therapy plays an important role in reducing the mortality associated with this condition. On the other hand ambiguous culture results lead to diagnostic uncertainty in clinical management and are associated with increased health care costs due to unnecessary treatment and testing.

\section{Recommendations}

- Proper selection of patients

- Assemble all necessary materials

- To use appropriate skin disinfectants, $70 \%$ alcohol and allowed to air dry and followed

- by $10 \%$ povidone-iodine and allow adequate contact time (2 minutes) with correct

- centrifugal cleaning technique.

- Avoid re-palpating the vein after skin disinfection

- Obtain adequate volume of blood depending on whether conventional manual bottles or

- commercial bottles for automated blood culture system is used

- Rubber tops of the blood culture bottles to be wiped with 70\% alcohol after the removal

- of protective cap

- Mix the blood thoroughly with the culture medium

- If any delay is anticipated to store the blood culture bottle at room temperature and

- avoid refrigeration

- Clearly label the blood culture bottle and furnish relevant patient and clinical details

- Avoid using canula or Central venous lines to obtain blood samples for blood culture

- To obtain at least two sets of blood cultures prior to the commencement of antibiotics

- Proper disposal of sharps

\section{References}

1. Washington, J. A. 1975. Blood cultures: principles and techniques. Mayo Clin. Proc. 50:91-97.

2. Richter, S. S., S. E. Beekmann, J. L. Croco, D. J. Diekema, F. P. Koontz, M. A. Pfaller, and G. V. Doern. 2002. Minimizing the workup of blood culture contaminants: implementation and evaluation of a laboratory-based algorithm. J. Clin. Microbiol. 40:2437-2444. 
3. Bates, D. W., L. Goldman, and T. H. Lee. 1991. Contaminant blood cultures and resource utilization: the true consequences of false-positive results. JAMA 265:365-369.

4. Archer, G. L. 1985. Coagulase-negative staphylococci in blood cultures: the clinician's dilemma. Infect. Control 6:477-478.

5. Kim S-D., L. C. McDonald, W. R. Jarvis, S. K. McAllister, R. Jerris, L. A. Carson, and J. M. Miller. 2000. Determining the significance of coagulase-negative staphylococci isolated from blood cultures at a community hospital: a role for species and strain identification. Infect. Control Hosp. Epidemiol. 21:213-217.

6. Kirchhoff, L. V., and J. N. Sheagren. 1985. Epidemiology and clinical significance of blood cultures positive for coagulase-negative staphylococcus. Infect. Control 6:479-486.

7. Updated Review of Blood Culture Contamination. Keri K. Hall and Jason A. Lyman Clin. Microbiol. Rev. October 2006 19: 788-802.

8. MacGregor, R. R., and H. N. Beaty. 1972. Evaluation of positive blood cultures. Guidelines for early differentiation of contaminated from valid positive cultures. Arch. Intern. Med. 130:84-87.

9. Weinstein, M. P., L. B. Reller, J. R. Murphy, and K. A. Lichtenstein. 1983. The clinical significance of positive blood cultures: a comprehensive analysis of 500 episodes of bacteremia and fungemia in adults. I. Laboratory and epidemiologic observations. Rev. Infect. Dis. 5:35-53.

10. Weinstein, M. P., M. L. Towns, S. M. Quartey, S. Mirrett, L. G. Reimer, G. Parmagiani, and L. B. Reller. 1997. The clinical significance of positive blood cultures in the 1990s: a prospective comprehensive evaluation of the microbiology, epidemiology, and outcome of bacteremia and fungemia in adults. Clin. Infect. Dis. 24:584-602.

11. Weinstein, M. P. 2003. Blood culture contamination: persisting problems and partial progress. J. Clin. Microbiol. 41:2275-2278.
12. Rupp, M. E., and G. L. Archer. 1994. Coagulase-negative staphylococci: pathogens associated with medical progress. Clin. Infect. Dis. 19:231-243.

13. Khatib, R., K. M. Riederer, J. A. Clarck, S. Khatib, L. E. Briski, and F. M. Wilson. 1995. Coagulase-negative staphylococci in multiple blood cultures: strain relatedness and determinants of samestrain bacteremia. J. Clin. Microbiol. 33:816-820.

14. Schifman, R. B., C. L. Strand, F. A. Meier, and P. J. Howanitz. 1998. Blood culture contamination: a College of American Pathologists Q-Probes study involving 640 institutions and 497134 specimens from adult patients. Arch. Pathol. Lab. Med. 122:216-221.

15. Bates, D. W., and T. H. Lee. 1992. Rapid classification of positive blood cultures: prospective validation of a multivariate algorithm. JAMA 267:1962-1966.

16. Mylotte, J. M., and A. Tayara. 2000. Blood cultures: clinical aspects and controversies. Eur. J. Clin. Microbiol. Infect. Dis. 19:157-163.

17. Reimer, L. G., M. L. Wilson, and M. P. Weinstein. 1997. Update on detection of bacteremia and fungemia. Clin. Microbiol. Rev. 10:444-465.

18. Souvenir, D., D. E. Anderson, Jr., S. Palpant, H. Mroch, S. Askin, J. Anderson, J. Claridge, J. Eiland, C. Malone, M. W. Garrison, P. Watson, and D. M. Campbell. 1998. Blood cultures positive for coagulase-negative staphylococci: antisepsis, pseudobacteremia, and therapy of patients. J. Clin. Microbiol. 36:1923-1926.

19. Shafazand, S., and A. B. Weinacker. 2002. Blood cultures in the critical care unit: improving utilization and yield. Chest. 122:1727-736.

20. Herwaldt, L. A., M. Geiss, C. Kao, and M. A. Pfaller. 1996. The positive predictive value of isolating coagulase-negative staphylococci from blood cultures. Clin. Infect. Dis. 22:14-20.

21. Beekmann, S. E., D. J. Diekema, and G. V. Doern. 2005. Determining the clinical significance of coagulase-negative staphylococci isolated from blood cultures. Infect. Control Hosp. Epidemiol. 26:559-566. 
22. Weinstein, M. P., L. B. Reller, J. R. Murphy, and K. A. Lichtenstein. 1983. The clinical significance of positive blood cultures: a comprehensive analysis of 500 episodes of bacteremia and fungemia in adults. I. Laboratory and epidemiologic observations. Rev. Infect. Dis. 5:35-53.

23. Aronson, M. D., and D. H. Bor. 1987. Blood cultures. Ann. Intern. Med. 106:246-253.[Abstract/Free Full Text]

24. Chandrasekar, P. H., and W. J. Brown. 1994. Clinical issues of blood cultures. Arch. Intern. Med. 154:841-849.

25. Mirrett, S., M. P. Weinstein, L. G. Reimer, M. L. Wilson, and L. B. Reller. 2001. Relevance of the number of positive bottles in determining clinical significance of coagulase-negative staphylococci in blood cultures. J. Clin. Microbiol. 39:3279-3281.

26. Peacock, S. J., I. C. Bowler, and D. W. Crook. 1995. Positive predictive value of blood cultures growing coagulasenegative staphylococci. Lancet 346:191-192.

27. Everts, R. J., E. N. Vinson, P. O. Adholla, and L. B. Reller. 2001. Contamination of catheter-drawn blood cultures. J. Clin. Microbiol. 39:3393-3394.

28. Hardy, D. J., B. B. Hulbert, and P. C. Migneault. 1992. Time to detection of positive BacT/Alert blood cultures and lack of need for routine subculture of 5to 7-day negative cultures. J. Clin. Microbiol. 30:2743-745.

29. Huang, A. H., J. J. Yan, and J. J. Wu. 1998. Comparison of five days versus seven days of incubation for detection of positive blood cultures by the Bactec 9240 system. Eur. J. Clin. Microbiol. Infect. Dis. 17:637-641.

30. Haimi-Cohen, Y., S. Shafinoori, V. Tucci, and L. G. Rubin. 2003. Use of incubation time to detection in BACTEC 9240 to distinguish coagulase-negative staphylococcal contamination from infection in pediatric blood cultures. Pediatr. Infect. Dis. J. 22:968-974.

31. Lyytikainen, O., V. Valtonen, V. J. Anttila, and P. Ruutu. 1998. Evaluation of clinical and laboratory findings in leukaemic patients with blood cultures positive for Staphylococcus epidermidis. J. Hosp. Infect. 38:27.

32. Berger, C., J. Uehlinger, D. Ghelfi, N. Blau, and S. Fanconi. 1995. Comparison of C-reactive protein and white blood cell count with differential in neonates at risk for septicaemia. Eur. J. Pediatr. 154:138-144.

33. Pourcyrous, M., H. S. Bada, S. B. Korones, V. Baselski, and S. P. Wong. 1993. Significance of serial C-reactive protein responses in neonatal infection and other disorders. Pediatrics 92:431435.

34. Rubin, L. G., P. J. Sanchez, J. Siegel, G. Levine, L. Saiman, W. R. Jarvis, et al. 2002. Evaluation and treatment of neonates with suspected late-onset sepsis: a survey of neonatologists' practices. Pediatrics 110:e42.

35. Santolaya, M. E., J. Cofre, and V. Beresi. 1994. C-reactive protein: a valuable aid for the management of febrile children with cancer and neutropenia. Clin. Infect. Dis. 18:589-595.

36. Gowardman, J. R., C. Montgomery, S. Thirlwell, J. Shewan, A. Idema, P. D. Larsen, and J. H. Havill. 1998. Central venous catheter-related bloodstream infections: an analysis of incidence and risk factors in a cohort of 400 patients. Intensive Care Med. 24:1034-1039.

37. Tacconelli, E., M. Tumbarello, M. Pittiruti, F. Leone, M. B. Lucia, R. Cauda, and L. Ortona. 1997. Central venous catheter-related sepsis in a cohort of 366 hospitalised patients. Eur. J. Clin. Microbiol. Infect. Dis. 16:203-209.

38. Beutz, M., G. Sherman, J. Mayfield, V. J. Fraser, and M. H. Kollef. 2003. Clinical utility of blood cultures drawn from central vein catheters and peripheral venipuncture in critically ill medical patients. Chest 123:854-861.

39. Everts, R. J., E. N. Vinson, P. O. Adholla, and L. B. Reller. 2001. Contamination of catheter-drawn blood cultures. J. Clin. Microbiol. 39:3393-3394.

40. McBryde, E. S., M. Tilse, and J. McCormack. 2005. Comparison of contamination rates of catheter-drawn and peripheral blood cultures. J. Hosp. Infect. 60:118-121. 
41. Tokars, J. I. 2004. Predictive value of blood cultures positive for coagulasenegative staphylococci: implications for patient care and health care quality assurance. Clin. Infect. Dis. 39:333-341.

42. Brown, E., R. P. Wenzel, and J. 0. Hendley. 1989. Exploration of the microbial anatomy of normal human skin by using plasmid profiles of coagulase-negative staphylococci: search for the reservoir of resident skin flora. J. Infect. Dis. 160:644-650.

43. Selwyn, S., and H. Ellis. 1972. Skin bacteria and skin disinfection reconsidered. Br. Med. J. i:136-140.

44. Chapnick, E. K., B. C. Schaffer, J. D. Gradon, L. I. Lutwick, S. A. Krigsman, and M. Levi. 1991. Technique for drawing blood for cultures: is changing needles truly necessary? Southern Med. J. 84:1197-1198.

45. Murray, P. R., P. Traynor, and D. Hopson. 1992. Critical assessment of blood culture techniques: analysis of recovery of obligate and facultative anaerobes, strict aerobic bacteria, and fungi in aerobic and anaerobic blood culture bottles. J. Clin. Microbiol. 30:1462-1468.

46. Mimoz, O., A. Karim, A. Mercat, M. Cosseron, B. Falissard, F. Parker, C. Richard, K. Samii, and P. Nordmann. 1999. Chlorhexidine compared with povidone-iodine as skin preparation before blood culture. A randomized, controlled trial. Ann. Intern. Med. 131:834-837.

47. Barenfanger, J., C. Drake, J. Lawhorn, and S. J. Verhulst. 2004. Comparison of chlorhexidine and tincture of iodine for skin antisepsis in preparation for blood sample collection. J. Clin. Microbiol. 42:2216-2217.

48. Trautner, B. W., J. E. Clarridge, and R. O. Darouiche. 2002. Skin antisepsis kits containing alcohol and chlorhexidine gluconate or tincture of iodine are associated with low rates of blood culture contamination. Infect. Control Hosp. Epidemiol. 23:397-401.

49. Little, J. R., P. R. Murray, P. S. Traynor, and E. Spitznagel. 1999. A randomized trial of povidone-iodine compared with iodine tincture for venepuncture site disinfection: effects on rates of blood culture contamination. Am. J. Med. 107:119-125.

50. Strand, C. L., R. R. Wajsbort, and K. Sturmann. 1993. Effect of iodophor vs iodine tincture skin preparation on blood culture contamination rate. JAMA 269:1004-1006.

51. Calfee, D. P., and B. M. Farr. 2002. Comparison of four antiseptic preparations for skin in the prevention of contamination of percutaneously drawn blood cultures: a randomized trial. J. Clin. Microbiol. 40:1660-1665.

52. Isaacman, D. J., and R. B. Karasic. 1990. Lack of effect of changing needles on contamination of blood cultures. Pediatr. Infect. Dis. J. 9:274-278.

53. Krumholz, H. M., S. Cummings, and M. York. 1990. Blood culture phlebotomy: switching needles does not prevent contamination. Ann. Intern. Med. 113:290-292. (Erratum, 113:723.)

54. Spitalnic, S. J., R. H. Woolard, and L. A. Mermel. 1995. The significance of changing needles when inoculating blood cultures: a meta-analysis. Clin. Infect. Dis. 21:1103-1106.

55. Surdulescu, S., D. Utamsingh, and R. Shekar. 1998. Phlebotomy teams reduce blood-culture contamination rate and save money. Clin. Perform. Qual. Health Care 6:60-62.

56. Weinbaum, F. I., S. Lavie, M. Danek, D. Sixsmith, G. F. Heinrich, and S. S. Mills. 1997. Doing it right the first time: quality improvement and the contaminant blood culture. J. Clin. Microbiol. 35:563-565.

57. Schifman, R. B., P. Bachner, and P. J. Howanitz. 1996. Blood culture quality improvement: a College of American Pathologists Q-Probes study involving 909 institutions and 289572 blood culture sets. Arch. Pathol. Lab. Med. 120:999-1002.

58. Weinbaum, F. I., S. Lavie, M. Danek, D. Sixsmith, G. F. Heinrich, and S. S. Mills. 1997. Doing it right the first time: quality improvement and the contaminant blood culture. J. Clin. Microbiol. 35:563-565.

59. Gibb, A. P., B. Hill, B. Chorel, and R. Brant. 1997. Reduction in blood culture 
contamination rate by feedback to phlebotomists. Arch. Pathol. Lab. Med. 121:503-507.

60. Chinniah T R. 2007. Automated blood culture system - A Panacea, an asset or a liability? An audit at James Paget University Hospital. Abstract OP 11. 16th Annual. Scientific Sessions. Sri Lanka College of microbiologists, Sri Lanka

61. Leibovici, L., S. Greenshtain, O. Cohen, F. Mor, and A. J. Wysenbeek. 1991. Bacteremia in febrile patients. A clinical model for diagnosis. Arch. Intern. Med. 151:1801-1806.

62. Bennett, I L., Beeson, R B,. 1954. Bacteramia:A consideration of some experimental and clinical aspects. Yale J Biol Med. 262: 242 - 262

63. Thomson, R. B., C. Corbin, and J. S. Tan. 1989. Timing of blood culture collection from febrile patients, abstr. C-227, p. 431. Abstr. 89th Annu. Meet. Am. Soc. Microbiol. 1989. American Society for Microbiology, Washington, DC.

64. Stefan Riedel,1 Paul Bourbeau,2 Brandi Swartz,2 Steven Brecher et al. 2008. Timing of Specimen Collection for Blood Cultures from Febrile Patients with Bacteremia. J Clin Micro. 46: 13811385

65. Ilstrup D. M. Washington, J. A., II. 1983. The importance of volume of blood culture in the detection of bacteremia and fungemia. Diag Microbiol Infect Dis. 1: $107-110$

66. Eyster E, Bernene J. 1973. Nosocomial anaemia. JAMA. 223: 73 - 74

67. Durbin DA., Szymaczak EG., Goldman DA. 1970. Quantitative blood culture in childhood bacteremia. J Paeditr. 92: $778-780$. 